\title{
Etude faunistique des Chironomidés (Diptera) de la Haute Neste d'Aure (Pyrénées Centrales) : impact des aménagements hydroélectriques
}

\author{
G. Gazagnes! \\ H. Laville1
}

Mots clés : Diptera, Chironomidae, Eaux courantes, Faunistique, Ecologie, Impact humain.

Cette étude concerne les Chironomidés du réseau hydrographique de la Neste d'Aure, torrent pyrénéen de moyenne al titude $(1000$ à $1500 \mathrm{~m}$ ). Les récoltes faites par dérive dans huit stations, en été (août et septembre 1980) et en hiver (février 1981), permettent de caractériser le peuplement d'un torrent de moyenne altitude qui est comparé à celui vivant dans l'Estaragne situé dans la partie haute du réseau $(2370-1850 \mathrm{~m})$.

La comparaison des peuplements entre l'amont et l'aval de trois retenues hydroélectriques montre l'importance des débits réservés pour le maintien des écosystèmes originaux.

A faunistic study of the chironomids (Diptera) of the upper Neste d'Aure (Central Pyrenees) : the impact of hydroelectric schemes

Keywords : Diptera, Chironomidae, Running waters, Faunistic, Ecology, Human impact.

This is a study of the chironomids of the hydrographical system of the Neste d'Aure, a Pyrenean river at mean altitude (1000 to $1500 \mathrm{~m}$ ). Drift samples taken at eight stations in summer (August and September 1980) and winter (February 1981) were used to characterise the population of the river at mean altitude and were compared with the population in Estaragne, a river in the same system but at higher altitude $(2370 \cdot 1850 \mathrm{~m})$.

A comparison of populations upstream and downstream of three reservoirs showed the importance of flow reserves for maintaining the original ecosystem.

\section{Introduction}

Cette étude concerne les Chironomidés du réseau hydrographique de la Neste d'Aure (entre $1100 \mathrm{~m}$ et $1500 \mathrm{~m}$ d'altitude) dans les Pyrénées Centrales. Issue de recherches consacrées à l'ensemble de la faune macroinvertébrée (Gazagnes 1983) elle était destinée à compléter nos connaissances sur la distribution

1. Laboratoire d'Hydrobiologie, UA 695 CNRS, Université Paul Sabatier, 118, route de Narbonne, 31062 Toulouse Cedex, France. Cette étude a fait l'objet d'une communication au XXIX ${ }^{*}$ Congrès de l'Association Française de Limmologie qui s'est tenu à Toulouse du 29 au 3! Mai 1985. altitudinale des espèces dans les Pyrénées à la suite du travail réalisé antérieu rement (Laville \& Lavandier 1977) dans le torrent d'Estaragne situé dans la partie haute de ce même réseau hydrographique (fig.1). L'existence de 3 retenues dans la zone étudiée nous a en outre amené à apprécier les conséquences des modificat ions artificielles de débits par comparaison des peuplements chironomidiens à l'amont et à l'aval de ces retenues hydroélectriques. S'il est connu que les Chironomidés sont, avec les Oligochètes et les Amphiprodes, les invertébrés qui résistent le mieux aux $m$ difications de débits à l'aval des barrages (Ward $\&$ Stanfort 1979) il y a peu d'informations disponibles ur l'écologie des rivières 


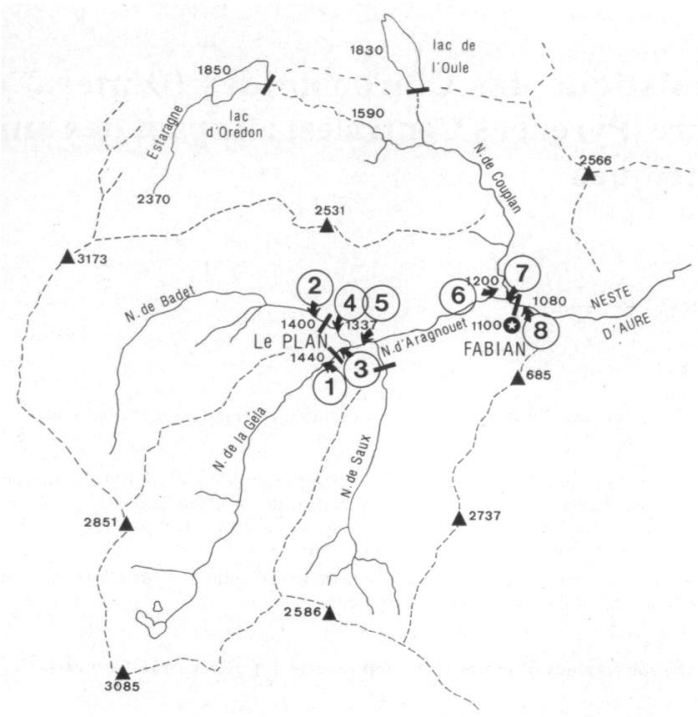

Fig. 1. Réseau hydrographique des Nestes de la Haute vallée d'Aure: emplacements des 8 stations d'étude.

présentant une réduction drastique du débit (Lillehammer \& Saltveit 1979). Baxter (1977) a recensé les différents effets de l'implantation des barrages sur la portion aval des cours d'eau. Ward \& Stanford (1979) ont passé en revue les divers impacts de la régulation des cours d'eau sur le zoobenthos.

En France, ce problème d'impact des ouvrages n'a été abordé que récemment sur de grands barrages des rivières du Sud-Ouest : le Lot, la Truyère et la Dordogne (Décamps et al. 1979) ou sur ceux du Verdon dans les Alpes de Haute Provence (Grégoire 1981). Chauvet (1983) a étudié les effets d'une réduc. tion de débit sur les invertébrés et les Salmonidés d'un torrent pyrénéen, l'Aston (1 $200 \mathrm{~m}$ ) et.posé le problème des débits réservés et de leur régularité.

La comparaison des populations de Chironomidés des tronçons de rivières à débit naturel avec celles des tronçons aménagés pourra servir de références scientifiques à ce problème de débits réservés.

\section{1. - Méthodes et stations d'étude}

L'échantillonnage a été effectué à l'aide de filets à dérive d'entrée carrée de $0.125 \mathrm{~m}$ de côté, de $1.20 \mathrm{~m}$ de long et de $240 \mu$ de vide de maille. Un deuxieme filet, fait d'un treillis plastifiè à mailles de $1 \mathrm{~cm}$, est emboité dans le premier filet dans le but de recueil. lir les feuilles mortes, brindilles et graviers et d'éviter ainsi un trop rapide colmatage.

Trois séries d'échantillonage - août 1980, septembre 1980, février 1981 - ont été effectuées sur un cycle de $24 \mathrm{~h}$ avec une périodicité de $3 \mathrm{~h}$. Les filets étaient entièrement immergés dans les zones de plus fort débit mais dont la profondeur permettait de filtrer toute la colonne d'eau.

Huit stations à débit naturel ou régulé ont été choisies sur le réseau hydrographique des Nestes de la Géla, du Badet, d'Aragnouet, de Couplan et d'Aure (fig. 1). Elles se situent entre l'étage montagnard 
(1800-1300 m) où dominent le Hêtre, le Sapin et le Bouleau et l'étage collinéen $(<1300 \mathrm{~m})$ avec la série du Chêne sessile associé à des landes à Buis et Noisetiers. Toutes, sauf la station 2 , se situent dans des vallées très encaissées et ombragées.

Les Nestes s'écoulent sur des moraines glaciaires 't des alluvions modernes qui recouvrent des schistes calcaireux et des grès du Dévonien, auréole métamorphique du massif granitique du Néouvielle.

Jusqu'à Fabian $(1100 \mathrm{~m})$, le régime des Nestes est de type nival à nival de transition -fonte des neiges de mai à juillet, pluies d'octobre à novembre- et torrentiel. Les débits ont été mesurés mensuellement de mai 81 à avril 82 . Leur évolution annuelle dans trois stations $(2,4,8)$ présentant un régime différent est reproduite sur la figure 2 .

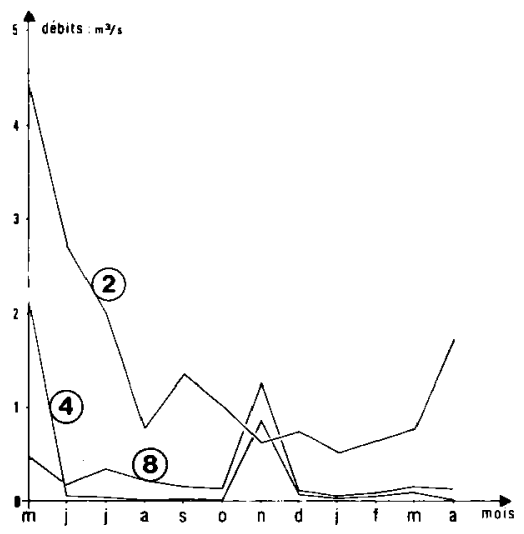

Fig. 2. Evolution annuelle des débits $(\mathrm{m} 3 / \mathrm{s})$ dans les stations 2 (non régulée), 4 (régulée sans débit réservé) et 8 (régulée à débit réservé) : mesures mensuelles.

- Les débits des stations 1 et 2, à l'amont des retenues, sont naturels. Les stations 3,4 et 5 , situées à l'aval de deux retenues n'ont pas de débit réservé ; l'eau qui s'écoule en été provient des fuites de vannes des prises d'eau et des sources et ruisselets du bassin versant : ses débits sont particulièrement faibles ( 4 et $15 \mathrm{l} / \mathrm{s}$ ).
- Le débit de la station 6 est réduit car les eaux originelles d'alimentation sont captées dans les hauts barrages du Massif de Néouvielle (Aumar. Aubert, Capdelong). L'alimentation provient surtout des sources d'Artigusse $(1590 \mathrm{~m})$.

- Le débit de la station 7 résulte des faibles débits de la Neste d'A ragnouet et des débits relativement constants de la Neste de Couplan.

La station 8, à l'aval de la retenue de Fabian, est la seule à bénéficier d'un débit réservé (125 V/s).

Pendant les crues, toutes ces stations peuvent retrouver leurs lits et leurs débits d'origine.

Les principales caractéristiques physiques altitude, largeur du lit et débits d'étiage et de crue, températures maxima/minima, résistivité- et chimique-dureté calcique-sont récapitulées sur le tableau I.

En période d'étiage, les faibles résistivités mesurées aux stations 3,4 et 5 traduisent une très forte minéralisation, alors qu'en période de crue elles s'uniformisent avec celles des autres stations. Il en est de mème pour les autres paramètres chimiques qui traduisent cette concentration des éléments dissous. A la station 8 , par contre, l'installation d'un débit réservé atténue cet effet de concentration constaté aux stations 3, 4 et 5 .

Sur l'ensemble du réseau étudié, le substrat est comparable et constitué de blocs, de galets et graviers avec une couverture hivernale de périphyton à base d'hydrures et de Diatomées.

\section{2. - Les Chironomidés de la Haute Neste d'Aure}

\subsection{Etude faunistique et écologique}

La liste faunistique des huit stations d'étude est récapitulée sur le tableau II.

65 taxons de la famille des Chironomidae, pour la plupart identifiés à l'espèce, ont été dénombrés. Ils se répartissent ainsi dans les principales sousfamilles ou tribus : 4 Tanypodinae, 8 Diamesinae, 43 Orthocladiinae, 3 Chironomini, 7 Tanytarsini.

La quasi-totalité des espèces est essentiellement rhéophile. Le peuplement mis en évidence sur la 
Tableau I. Principales caractêristiques physico-chimiques des 8 stations de la Haute Neste d'Aure. E : étiage ; C : crue.

\begin{tabular}{|c|c|c|c|c|c|c|c|c|c|c|c|}
\hline \multirow{2}{*}{ STATIONS } & \multirow{2}{*}{$\begin{array}{c}\text { ALTITLDES } \\
\mathrm{m}\end{array}$} & \multicolumn{2}{|c|}{$\begin{array}{c}\text { LARGEUR OU LIT } \\
\mathrm{m}\end{array}$} & \multicolumn{2}{|c|}{$\begin{array}{c}\text { DEBIT INSTANTANE } \\
\mathrm{m} 3 / \mathrm{s}\end{array}$} & \multicolumn{2}{|c|}{$\begin{array}{l}\text { TEMPERATURES } \\
{ }^{\circ} \mathrm{C} \text {. }\end{array}$} & \multicolumn{2}{|c|}{$\begin{array}{l}\text { RESISTIVITE } \\
/ \mathrm{cm} 2 / \mathrm{CT}\end{array}$} & \multicolumn{2}{|c|}{$\begin{array}{c}\text { DURETE CALCLOUE } \\
\text { MgII } C_{2} O_{3}\end{array}$} \\
\hline & & $E$ & $c$ & E & c & $\operatorname{Max}$. & min. & Max. & Min. & Max. & $H_{i n}$. \\
\hline 1 & 1440 & 4 & 5 & 0.3 & 2,4 & 10,9 & 1 & 7820 & 5954 & 75 & 64 \\
\hline 2 & 1400 & 3 & 12 & 0,5 & 4,4 & 11,1 & 1 & 8425 & 6899 & 66 & 56 \\
\hline 3 & 1337 & 0,5 & 4 & 0,004 & 0,4 & 11 & 1 & 6941 & 3914 & 114 & 79 \\
\hline 4 & 1337 & 1,25 & 4,5 & 0,011 & 0,9 & 14,2 & 0,5 & 7873 & 5. 050 & 89 & 60 \\
\hline 5 & 1337 & 1 & 7 & 0,015 & 1,3 & 12 & 0,5 & 7919 & 4558 & 93 & $=9$ \\
\hline 6 & 1200 & 6 & 6,5 & 0,3 & 1,1 & 13,9 & 0,5 & 9698 & 8041 & 62 & 40 \\
\hline 7 & 1100 & 4,5 & 9 & 0,4 & 1,9 & 12,8 & 1,2 & 8435 & 6790 & 72 & 52 \\
\hline 8 & 1080 & 16 & 3,5 & 0.125 & 1.25 & 11 & 1,2 & 8199 & 6772 & 70 & 54 \\
\hline
\end{tabular}

Haute Neste d'Aure et ses affluents diffère de celuj du torrent d'Estaragne, situé dans l'étage subalpin $(1800 \mathrm{~m}-2300 \mathrm{~m})$ et alpin ( $2300 \mathrm{~m}$ ), essentiellement par la diminution de la diversité des Diamesinae (Tableau III).

Tableau III. Comparaison des peuplements chironomidiens du torrent d'Estaragne (Laville \& Lavandier 1977) et de la Haute Neste d'Aure.

\begin{tabular}{|c|c|c|c|c|}
\hline & \multicolumn{2}{|c|}{ ESTARAGME } & \multicolumn{2}{|c|}{ HRUTE MESTE D'AURE } \\
\hline & $*$ & s & N & * \\
\hline Tanypad inae & 3 & 5,2 & 4 & 6.1 \\
\hline Diames tnae & 11 & 18,9 & 8 & 12,3 \\
\hline Orthoc ladi inae & $3 B$ & 65,5 & 43 & 66,2 \\
\hline Chironoentini & 1 & 1,7 & 3 & 4,6 \\
\hline Tarytarsini & 5 & 8,6 & 7 & 10.8 \\
\hline TOTAL & 58 & & 65 & \\
\hline
\end{tabular}

Les températures plus froides de l'Estaragne sont favorables aux représentants de cette sous-famille qui colonisent de préférence les milieux froids de haute montagne: en été, les températures des sta. tions les plus froides de l'Estaragne ne dépassent pas $8,5^{\circ} \mathrm{C}$, celles de la Neste d'Aure peuvent atteindre $11^{\circ}$ à $14^{\circ} \mathrm{C}$.

La composition des deux peuplements au niveau spécifique est récapitulée sur le tableau IV qui regroupe les espèces recensées uniquement dans l'un ou l'autre torrent et les espèces communes aux deux.

\section{DIAMESINAE}

Sur les 8 Diamesinae identifiés dans la Haute Neste d'Aure, 3 n'avaient pas été récoltées dans l'Estaragne.

- Diamesa vaillanti

- Boreoheptagia legeri

- Diamesa gr. dampit.

Cinq espèces de l'Estaragne n'ont pas été retrouvées dans les dérives de la Neste : trois d'entre elles, boréoalpines ou alpines, n'ont jusqu'ici jamais été signalées au-dessous de $1450 \mathrm{~m}$ dans les Pyrénées.

- Diamesa laticauda

- Diamesa wuelkeri

- Pseudodiamesa branickii

Les deux autres, à caractère moins psychrotherme, Diamesa cinerella et Pseudokiefferiella parva sont pourtant signalées des Pyrénées à des altitudes comparables ou même inférieures à celles de nos stations.

En 1983, nous avons récolté Diamesa zemyi à 1000 m et Diamesa latitarsis à $610 \mathrm{~m}$ à l'aval du tronçon étudié de la Neste d'Aure. Dans les Alpes, D. latitarsis a son maximum de fréquence entre 800 et 1500 $\mathrm{m}$ : elle peut donc également caractériser ce torrent de moyenne montagne qu'est la Haute Neste d'Aure. 
Tableau II. Liste faunistique des Chironomidés récoltés dans 8 stations du réseau hydrographique de la Haute Neste d'Aure.

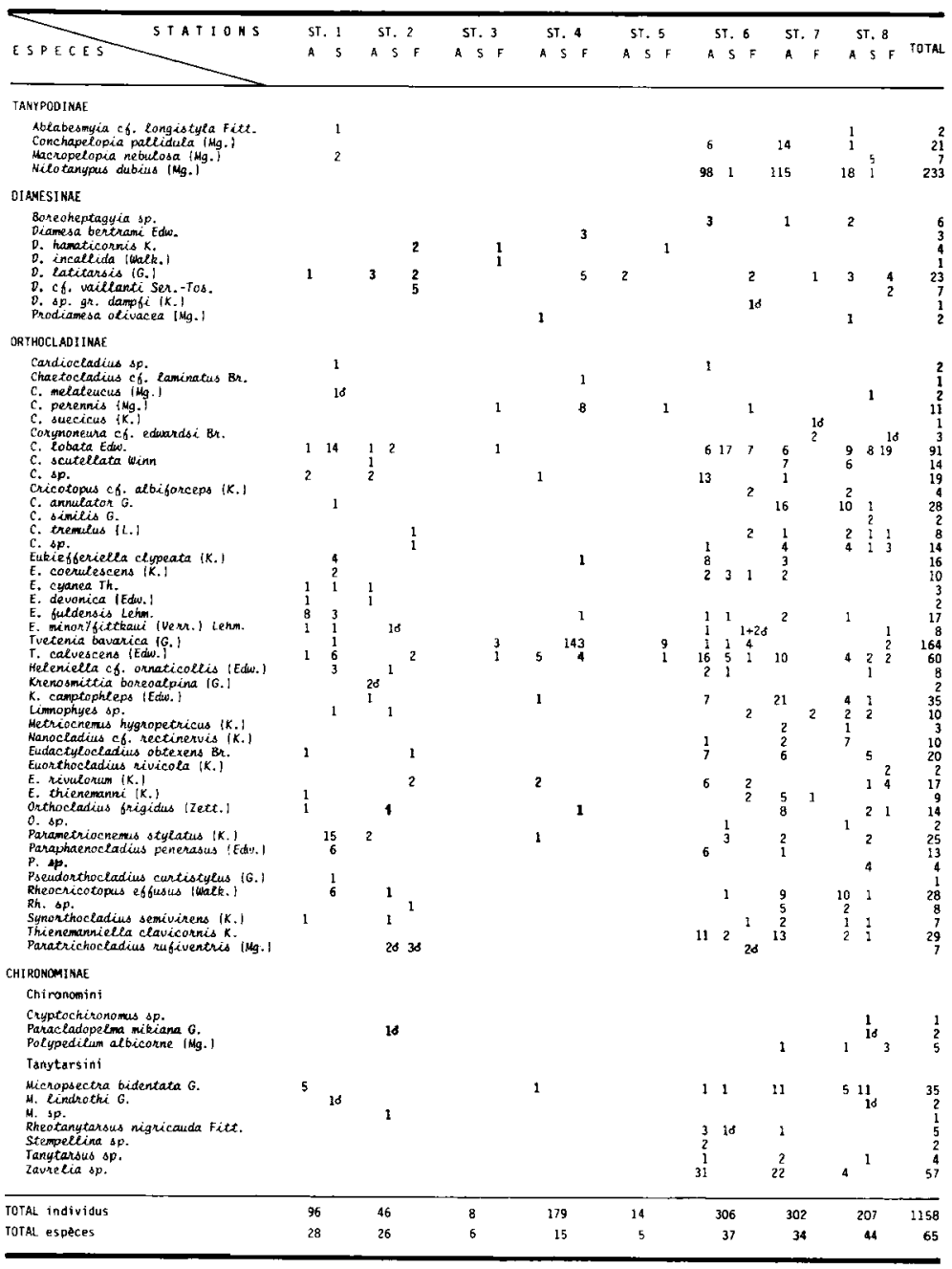


Tableau IV. Répartition altidudinale des principales espèces de Chironomidés de l'Estaragne (2 $370 \mathrm{~m})$ jusqu'à la Neste d'Aure (1 $100 \mathrm{~m})$.

\begin{tabular}{|c|c|c|}
\hline $\begin{array}{c}\text { ESTARAgne } \\
2370 m-1850 m\end{array}$ & $\begin{array}{l}\text { Espèces communes } \\
\text { ESTARAGNE + NESTE D'AURE }\end{array}$ & $\begin{array}{l}\text { NESTE D'AURE } \\
1500 \mathrm{~m}=1200 \mathrm{~m}\end{array}$ \\
\hline $\begin{array}{l}\text { Dianesa laticauda } \\
\text { Diamesa wïlkeri } \\
\text { Psevdokiefferiella branickii } \\
\text { Eukiefferiella fittkaui } \\
\text { Eukiefferieila tiralensis } \\
\text { Krenosmittia boreoalpina } \\
\text { Paratrichociadius skirwithensis } \\
\text { Krenopsectra fallax } \\
\text { Micropsectra coracina }\end{array}$ & $\begin{array}{l}\text { Diamesa hamaticornis } \\
\text { Diamesa latitarsis } \\
\text { Diamesa zernyi } \\
\text { Chaetocladius laminatus } \\
\text { Corynoneura lobata } \\
\text { Cricotopus annulator } \\
\text { Eukiefferiella coerulescens } \\
\text { Eukiefferiella devonica } \\
\text { Eukiefferiella fuldensis } \\
\text { Tvetenia bavarica } \\
\text { Tvetenia calvescens } \\
\text { Orthocladius frigidus } \\
\text { Orthocladius fuscimanus } \\
\text { Orthocladius rivicola } \\
\text { Paraphaenociadius penerasus } \\
\text { Paratrichociadius rufiventris } \\
\text { Rheocricotodus effusus } \\
\text { Polypedilum albicorne } \\
\text { Micropsectra bigentata }\end{array}$ & $\begin{array}{l}\text { Boreoheptagia legeri } \\
\text { Dianesa? vaillanti } \\
\text { Chaetocladius perennis } \\
\text { Cricotopus tremulus } \\
\text { Eukiefferiella minor } \\
\text { Eukiefferiella clypeata } \\
\text { Eukiefferiella cyanea }\end{array}$ \\
\hline
\end{tabular}

\section{ORTHOCLADIINAE}

Les Orthocladiinae sont moins diversifiés dans l'Estaragne ( 37 espèces) que dans la haute Neste d'Aure (44 espèces). Les différences entre les deux peuplements apparaissent mieux si on compare les espèces des 3 genres les plus diversifiés : Eukiefferiella + Tvetenia, Orthocladius, Cricotopus.

\section{Eukiefferiella et Tvetenia}

Deux Eukiefferiella, E. fitikaui et E. tirolensis, espèces psychrothermes de l'Estaragne n'ont pas été retrouvées dans la Haute Neste d'Aure. Bien que les nymphes de $E$. fittkaui et $E$. minor ne se différencient pas, trois imagos $\sigma$ de E. minor identifiés aux stations 2 et 6 montrent que les exuvies notées minor/fittkaui sur le tableau II appartiennent certainement à $E$. minor, réputée moins sténotherrne que $E$. fittkaui.

Eukiefferiella clypeata et E. cyanea n'ont pas été récoltées dans l'Estaragne. La première est très fréquente dans le Rhithral de la rivière Lot (Laville 1981) dans l'Epipotamal de l'Aveyron (ViaudChauvet 1982) ainsi que du Métharhithral au Potamal de la Fulda (Lehmann 1971). La seconde, n'avait pas été retrouvée dans les Pyrénées depuis les récoltes de Bertrand $(1950,1952)$ dans le Gave de Pau à Gavarnie (1300 m) et dans le cours espagnol du Rio Garona à Salardu $(1100 \mathrm{~m})$.

Elle a été récemment trouvée, assez abondante, dans le torrent pyrénéen, le Touyre $(650 \mathrm{~m})$, sousaffluent de la rivière Ariège.

Les deux Eukiefferiella, E. devonica et E. fuldensis rencontrées seulement dans les stations les plus chaudes de l'Estaragne semblent plus aptes à coloniser les torrents d'altitude moyenne.

E. devonica est signalée du Crénal à l'Hyporhithral de la Fulda (Lehmann 1971), dans tout le Rhithral du Lot (Laville 1981) et surtout dans l'Epipotamal de l'Aveyron (Viaud-Chauvet 1982). 
E. fuldensis colonise l'Epirhithral de la Fulda (Lehmann 1971); elle est plus fréquente dans le Crénal que dans l'Epirhithral du Lot (Laville 1981) mais elle n'apparaît que rarement dans l'Epi-et le Métapotamal de l'Aveyron (Viaud-Chauvet 1982). Dans les Alpes de Haute-Bavière, ses récoltes se situent vers $1450 \mathrm{~m}$ (Thienemann 1954). Elle est également signalée dans le cours moyen d'une rivière de la Forêt-Noire (Besch, Hoffmann \& Ellenberger 1967).

Cette espèce paraît donc moyennement psychrotherme et peut également caractériser le torrent de la Neste d'Aure.

Tvetenia bavarica n'était pas signalée dans l'Estaragne (Laville \& Lavandier 1977). Le matériel nymphal identifié Tvetenia calvescens a été revu et différencié au niveau des fourreaux alaires d'après Langton (1984). Trois stations de l'Estaragne échelonnées entre $1850 \mathrm{~m}$ et $2150 \mathrm{~m}$ ont révélé la présence de $T$. bavarica où ces deux espèces cohabitent.

\section{Orthocladius}

Orthocladius (Euorthocladius) rivulorum et $O$. (Euorthocladius) thienemanni apparaissent seulement vers $1400 \mathrm{~m}$ : ce sont deux espèces fréquentes dans les torrents de transition (Ubergangsbäche) des Alpes (Thienemann 1954). O. (Euorthocladius) rivicola vit depuis la source de l'Estaragne $(2400 \mathrm{~m})$ jusqu'à la dernière station aval de la Neste d'Aure et $O$. thienemanni seulement dans la Neste entre $1440 \mathrm{~m}$ et $610 \mathrm{~m}$. Les deux espèces coexistent entre $500 \mathrm{~m}$ et $1000 \mathrm{~m}$ dans un cours d'eau des HautTatras, où $O$. rivicola domine entre $850 \mathrm{~m}$ et 1000 met $O$. thienemanni entre $500 \mathrm{~m}$ et $800 \mathrm{~m}$ (Kawecka, Kownacka \& Kownachi 1971). On retrouve donc dans la Neste d'Aure une succession assez comparable à celle de ce torrent des Haut-Tatras.

\section{Cricotopus}

On observe un net accroissement de la diversité des Cricotopus dans la Haute Neste d'Aure. L'unique espèce de l'Estaragne inférieur $(1850 \mathrm{~m}) \mathrm{C}$. annulator parait à sa limite altitudinale. Dans un torrent des Alpes, l'Eau d'Olle, elle remonte jusqu'à $1900 \mathrm{~m}$ mais est une des espèces dominantes entre $700 \mathrm{~m}$ et $1200 \mathrm{~m}$ (Gay 1982). Outre C. anmulator, 3 Cricoropus, $C$. tremulus, $C$. similis et $C$. cf. albiforceps sont recensés dans la Neste entre $1100 \mathrm{~m}$ et $1400 \mathrm{~m} ; C$. tremulus et $C$. similis ont été récoltés en 1983 à 420 $m$ dans une station plus aval.
Krenosmittia borealpina colonise tout le cours de l'Estaragne et seulement la station 2 (1400 m) de la Neste d'Aure.

* Krenosmittia camptophleps cohabite avec $K$. borealpina à la station 2 mais devient plus abondante dans les stations aval. On peut considérer ces 2 espèces comme vicariantes : elles coexistent vers $1400 \mathrm{~m}$, sans doute au niveau de leurs limites respectives de répartition. $K$. camptophleps est citée pour la première fois dans les Pyrénées.

Patrichocladius rufiventris récoltée entre $2150 \mathrm{~m}$ et $610 \mathrm{~m}$ (en 1983) paraît relativement eurytherme. Lehmann (1971) la signale sur tout le cours de la Fulda. Elle est fréquente dans l'Hyporhithral du Lot (Laville 1981) mais plus rare dans l'Estaragne.

Paratrichocladius skimithensis, plus psychrotherme, prédomine dans les deux stations les plus froides de l'Estaragne : elle n'a jamais été récoltée au-dessous de $1850 \mathrm{~m}$. Dans les Alpes, elle est signalée à plus basse altitude, entre 1780 m et $725 \mathrm{~m}$ (Gay 1982). Dans l'Estaragne les 2 Paratrichocladius cohabitent entre $1850 \mathrm{~m}$ et $2150 \mathrm{~m}$.

\section{CHIRONOMINI}

* Paracladopelma mikiana (P. schlitzensis Ringe 1974, nov syn: Fittkau \& Reiss 1978) est nouvelle pour les Pyrénées. Signalée en France dans les Basses-Alpes près de Forcalquier à $500 \mathrm{~m}$ d'altitude c'est une espèce rhéophile polyoxybionte moyenne. ment sténotherme à eurytherme (Ringe 1974).

Polypedilum albicorne est le seul Chironomini de l'Estaragne. Il apparaît également dans les stations 7 et 8 de la Neste d'Aure et plus vers l'aval à Agos (780 m) (Laville 1980). Lehman (1971) le signale du Crénal à l'Epirhithral de la Fulda. Il peut être con. sidéré comme psychrotherme.

\section{TANYTARSINI}

Micropsectra coracina et Krenopsectra fallax sont deux espèces nettement sténothermes d'eau froide seulement récoltées dans l'Estaragne $(1850 \mathrm{~m})$.

Micropsectra bidentata paraît être moins sténotherme que $M$. coracina : elle est fréquente dans l'Estaragne ainsi que dans les deux stations inférieures (st. 7 et 8) de la Neste d'Aure. Lehmann (1971) l'a signalée du Crénal à l'Epirhithral de la Fulda.

Rheotanytarsus nigricauda est une espèce relativement sténotherme et caractéristique de l'Epirhithral du Lot et de la Fulda (Laville 1981). Dans la 
vallée d'Aure elle n'a jamais été récoltée au-dessus de $1200 \mathrm{~m}$.

Zavrelia? pentatoma est le Tanytarsini le plus fréquent des stations 6 à $8(1200 \mathrm{~m})$ de la Neste. Elle est signalée dans les Pyrénées atlantiques, dans la rivière Lissuraga, à plus basse altitude $(400 \mathrm{~m})$ (Laville 1980).

\subsection{Caractéristiques du peuplement}

Le peuplement chironomidien de la Haute Neste d'Aure peut être assimilé à celui d'un torrent de transition (Ưbergangsbach) défini par Thienemann (1954a) comme intermédiaire entre un torrent de haute montagne et une rivière de montagne moyenne (Mittelgebirgsbach) à Salmonidés.

- 3 espèces récoltées à plus de 90 exemplaires sont dominantes:

Nilotanypus dubius

Corynoneura lobata

Tvetenia bavarica

-11 espèces récoltées entre 20 et 60 exemplaires peuvent être considérées subdominantes :

Conchapelopia pallidula

Diamesa latitarsis

Cricotopus annulator

Eudactylocladius obtexens

Krenosmittia camptophleps

Parametriocnemus stylatus

Rheocricotopus effusus

Thienemanniella clavicomis

Tvetenia calvescens

Micropsectra bidentata

Zavrelia? pentatoma

- 10 espèces récoltées de 10 à 20 exemplaires sont fréquentes dans le peuplement

Chaetocladius perennis

Corynoneura scutellata

Eukiefferiella clypeata

Eukiefferiella coerulescens

Eukiefferiella fuldensis

Nanocladius? rectinervis

Euorthocladius rivulorum

Euorthocladius thienemanni

Orthocladius frigidus

Paraphaenocladius penerasus
Si l'on considère les stations à débit naturel : 1 , 2,6 et 7 , deux ensembles apparaissent sur le plan faunistique :

a) Nestes de la Géla et du Badet,

b) Nestes de Couplan et d'Aure.

a) Les Nestes de la Géla et du Badet sont caractérisées par une crue de printemps prolongée (avril à juillet) : le rapport des débits de mai à août $=8 / 1$. Les sources d'alimentation sont situées au niveau des étages subalpins et alpins ( $>1800 \mathrm{~m}$ ) et la température de l'eau ne dépasse $8^{\circ} \mathrm{C}$ que l'été (juin à septembre); l'amplitude thermique est de $10,5^{\circ} \mathrm{C}$.

b) Les sources de la Neste de Couplan sont dans l'étage montagnard ; la crue de printemps est plus faible : le rapport des débits de mai à août $=3,5 / 1$. La température est supérieure à $10^{\circ} \mathrm{C}$ de juin à septembre; elle atteint $14^{\circ} \mathrm{C}$ en août ; l'amplitude thermique est de $13,5^{\circ} \mathrm{C}$.

Sur la Neste d'Aure, en amont de la retenue de Fabian, la crue de printemps est également réduite : l'apport d'eau estival se limite à peu près à celui de la Neste de Couplan ; les eaux sont un peu plus froides par suite de la présence de sources.

Le plus faible nombre d'espèces de la Géla et du Badet peut traduire les crues plus violentes de ces Nestes : leur régime therrnique froid peut également favoriser l'installation d'espèces psychrothermes. Les Nestes d'Aure et de Couplan, aux températures plus élevées, permettent au contraire la colonisation d'espèces plus eurythermes de basse et moyenne vallée qui s'installent également du fait d'une plus grande stabilité du substrat.

C'est donc sur un fond de peuplement différent que s'exerce l'influence des aménagements hydroélectriques dans les stations 3,4 et 5 d'une part et dans la station 8 d'autre part.

\subsection{Affinités cénotiques entre les stations}

Le nombre total d'espèces de Chironomidés recensés dans les huit stations est récapitulé sur la figure 3.

Les peuplements des différentes stations ont été comparés deux à deux à l'aide de l'indice de similitude de $S_{\phi}$ rensen (1948):

$$
\text { IS }=\frac{2 c}{a+b}
$$

a et $b$ : nombre d'espèces de chaque station, c : nombre d'espèces communes aux deux stations. 


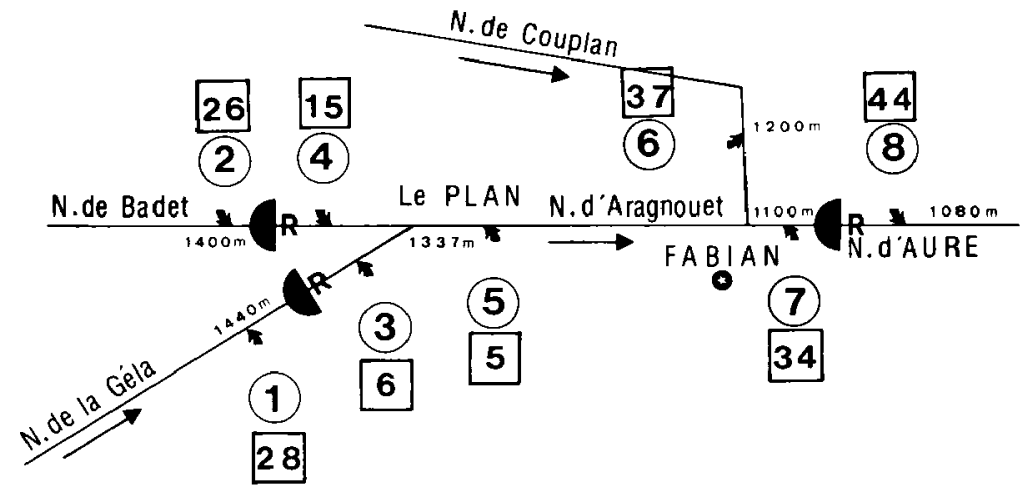

Fig.3. Nombre total d'espèces de Chironomidés recensés dans les 8 stations pour l'ensemble des trois séries d'échantilionnages. $\mathbf{R}$ : retenues.

Cet indice varie entre 0 (pas d'espèces communes) et 1 (même peuplement) : il permet d'évaluer le degré de ressemblance entre les peuplements des stations étudiées.

Nous avons calculé cet indice sur l'ensemble des dérives pour les huit stations (Tableau V) : on constate une affinité moyenne entre les stations 1 (Géla) et 2 (Badet), toutes deux situées à même altitude $(1400 \mathrm{~m})$ et à l'amont d'une retenue. Leur nombre d'espèces est comparable : 28 espèces à la st. 1 et 26 à la st. $2: 14$ espèces sont communes aux deux stations.

L'affinité des peuplements des stations 6,7 et 8 est plus forte $(0,64-0,73)$ de même que leur richesse spécifique.

A l'amont de la retenue de Fabian, les stations 6 (Couplan) avec 37 espèces et 7 (Aragnouet) avec 34 espèces ont 26 espèces communes et sont les plus affines.

La station 8 , bien que situee à l'aval de la retenue de Fabian, est la plus diversifiée (44 espèces); elle présente autant d'espèces communes (26-27) avec chacune des stations 6 et 7 . Le maintien d'un débit réservé, bien que faible $(125 \mathrm{l} / \mathrm{s})$, permet la conser. vation d'un peuplement chironomidien très diversifié ainsi que celui des Ephéméroptères et des
Tableau V. Comparaison des peuplements des stations deux à deux à l'aide de l'indice de Shrensen (1948) d'après la totalité des dérives.

\begin{tabular}{|c|c|c|c|c|c|c|c|}
\hline & 2 & 3 & 4 & 5 & 6 & 7 & 8 \\
\hline 1 & 0,52 & 0,17 & 0,42 & 0,18 & 0.55 & 0,55 & 0,55 \\
\hline 2 & & 0,18 & 0,34 & 0,19 & 0,50 & 0,50 & $0,5 \overline{4}$ \\
\hline 3 & & & 0,28 & & 0,18 & 0,20 & 0,12 \\
\hline 4 & & & & & 0,42 & 0,37 & 0,34 \\
\hline 5 & & & & & 0.19 & 0.10 & 0,12 \\
\hline 6 & & & & & & 0,73 & 0,64 \\
\hline 7 & & & & & & & 0.69 \\
\hline
\end{tabular}

Simulidés. Celui des Plécoptères et des Trichoptères tend à régresser.

Par contre, dans les stations intermédiaires 3, 4, 5 , soumises à des débits d'étiage particulièrement faibles (4 à $15 \mathrm{l} / \mathrm{s})$, on observe une nette diminution du nombre d'espèces de Chironomidés qui, en été, peut aller jusqu'à leur complète disparition : en août 0 espèce à la station 3,1 espèce, Diamesa latitarsis à la station 5 et 7 espèces à la station 4 .

La plupart des espèces (Diamesa, Tretenia, Corynoneura, Chaetocladius) recensées dans ces trois 
stations proviennent des récoltes de février. Les Chironomidés restent les représentants prédominants des stations 3 et 5 , par ailleurs particulièrement affines (IS $=0,72$ ). Les Plécoptères, les Ephéméroptères sauf Baetis alpinus Pictet et les Trichoptères ont pratiquement disparu des dérives des stations 3 à 5 .

\section{3. - Discussion}

Les changements dans la composition et l'abondance du zoobenthos à l'aval des retenues sont notamment attribués à plusieurs facteurs : aux conditions thermiques, aux modifications de débits et de substrat, ces dernières étant intimement liées.

Les variations de débits à l'aval de barrages hydroélectriques peuvent détruire les organismes benthiques et causer une réduction considérable de leur diversité (Fischer \& Lavoy 1972, Trotzky \& Gregory 1974). Elles peuvent également modifier la pro. ductivité des milieux aquatiques concernés : ainsi la biomasse peut-elle être, soit réduite, soit augmentée. A l'aval du barrage d'un réservoir de montagne du Colorado, la diversité était moindre que dans les rivières semblables non régulées mais la biomasse y était plus élevée (Ward 1974). Cet exemple illustre tout à fait le deuxième principe de Thienemann (1954b) selon lequel : " plus les conditions devient des conditions optimales, plus le nombre d'espèces diminue mais plus leur biomasse augmente".

Ward \& Short (1978) ont insisté sur la capacité des organismes benthiques à intégrer les conditions écologiques pour développer un système de classification préliminaire qui serait fondé sur la réponse des espèces à la régulation. Grégoire (1981) a choisi comme indicateurs biologiques de l'impact des barrages sur le Verdon trois groupes d'organismes : les Diatomées du périphyton, les Ephéméroptères et les Amphipodes. Les Chironomidés sont avec les Trichoptères filtreurs, les Amphipodes et les Oligochètes les groupes en augmentation, à l'aval des barrages (Ward \& Stanford 1974). Ils constituent $60 \%$ du peuplement macrobenthique à l'aval du barrage de l'Aston (Chauvet 1983). Dans ka Neste d'Aure, la diminution du nombre d'espèces peut être généralement associée à des fluctuations fréquentes de débits et inversernent, un peuplement riche en espèces est lié à des débits réguliers.
A l'aval des retenues, les Chironomidés constituent le groupe le plus permanent qui ne régresse qu'après les Plécoptères, les Ephéméroptères, les Trichoptères et les Simulidés. Leur forte dominance témoigne déjà d'une importante modification des conditions de milieu. C'est le groupe qui résiste le mieux à la réduction des débits. Il substis te jusqu'à un certain seuil qu'il doit être possible de déterminer à partir de l'évolution de sa diversité spécifique.

Cette hypothèse mériterait d'être testée à l'aval d'autres réservoirs avec des types de régulation de débits différents. Elle permettrait de recueillir des données scientifiques comparatives susceptibles de servir de critère pour un aménagement rationnel des cours d'eau préservant au maximum les communautés naturelles.

Travaux cltés

Baxter (R.M.). - Environmental effects of dams and impound. ments. Ann. Rev. Ecol. Syst., $8: 255-283$

Bertrand (H.). 1950, - Récoltes de Diptères Chironomides dans les Pyrenées. Vie et Milieu, 1 (3) : 345-355.

Bertrand (H.). 1952. - Récoltes de Diptères Chironomides dans les Pyrénées (2E note). Vie et Milieu, 3 (3) : 314-321.

Besch (W.), Hofmann (W.) \& Ellenberger (W.). 1967. - Das Mak. robenthos auf Polyäthylenerısubstraten in Fliessgewässern. 1. Die Kinzing, ein Fluss der unteren Saimoniden-und oberen Barben-Zone. Annls Limnol, 3 (3) : $331-368$.

Chauvet (R.). 1983. - Influence d'une réduction de débit sur un torrent de montagne: l'Aston (Ariège). Annls Limnol, 19 (1): 45.49 .

Décamps (H.), Capblancq (J.), Casanova (H.) \& Toureng (J.N.) 1979. - Hydrobiology of some regulated rivers in the south. west of France : pp 273-288. In : J.V. Ward \& J.A. Stanforl (Ed.) The ecology of regulated streams, New-York.

Fittkau (E.J.) \& Reiss (F.) 1978. - Chironomidae. In : Illies, J. (Ed.). Limnofauna Europaea. 2. Aufl, : 404440. G. Fisher, Stuttgart.

Fischer (S.G.) \& Lavoy (A.). 1972. - Differences in littoral fauna due to fluctuating water levels below a hydroelectric dam. $J$. Fish. Res. Bd Can., 29 : 1472-1476.

Gay (C.). 1982. - Les communautés benthiques d'un torrent des Alpes françaises l'Eau d'Olle (Isère). Trav. Lab. Hydrobiol, Grenoble, $71 / 73: 7-31$.

Gazagnes (G.) 1983. - Contribution à l'étude de la dérive des invertébrés sur la Haute Neste d'Aure; impact des aménage. ments hydrólectriques. Thèse 3e cycle, Hydrobiologic, Univ. P. Sabatier, Toulouse (France) : 104 p. +1 annexe

Grégoire (A.) 1981. - Contribution à l'étude hydrobiologique d'une rivière aménagée, le Verdon (Alpes de Haute-Provence). Les lacs de barrage et les tronçons de cours d'eau à débit régulé. Thèse Doctorat ès Sciences Naturelles. Univ. AixMarseille $1: 213 \mathrm{p}$

Kawecka (B.) Kownacka (M.) \& Kownacki (A.). 1971. - General characteristics of the biccoenosis in the streams of the Polish High Tatras. Acta Hydrobiol., 13 (4) : 465-476. 
L.ington (R.H.). 1984. - A key to pupal exuviae of British Chirotomidae: 324 p. P.H. Langton (Ed.). March, Cambridgeshire. Great Britain.

Latil]e (H.) 1980. - Inventaire 1980 des Chironomides (Diptera) connus des Pyrenees. Antls Limmol, 16 (3): $211-223$

Laville (H). 1981. - Récol tes d' exwies nymphales de Chironomides (Diptera) dans le Haut-Lut, de la source $(1295 \mathrm{~m})$, au confluent de la Truyère (223 m). Annls Limnol., 17 (3) : 255-289.

Laville (H.) \& Lavandier (P.). 1977. - Les Chironomides (Diptera) d'un torrent pyrenéen de haute montagne: l'Estaragne. Annls Limmol. 13 (1): 57.81 .

Lehıาasn (J.). 1971. - Die Chironomiden der Fulda (Systemat ische, okologische und faunistische Untersuchungen). Arch. Hydrobiol., Suppl. 37 : 466-555.

Lillehammer (A.) \& Saltveit (S.J.) 1979. - Stream regulation in Norway : pp 201-214. In : J.V. Ward \& J.A. Standorf (Ed.). The ecology of regulated streams. New-York.

Ringe $(F$, . 1974. - Chironomiden-Emergenz 1970 in Breitenbach und Rohrwiesenbach. Schlitzer Produktionsbiologische Studien (10). Arch. Hydrobiol., Suppl. 45 (2/3): 212-304.

S $\phi$ rensen (T.). 1948. - A method of establishing grisup of equal amplitude in plant sociology based on similarity of species content and its application to analyses of the vegetation on Danish commons. Biol $S k r$. $5: 1-34$.
Thienemann (A.). 1954a. - Chironomus. Leben, Verbreitung und wirtschatliche Bedeutung der Chironomiden. Binnengewäser 20: $834 \mathrm{P}$

Thienemans (A.). 1954b. - Ein drittes biozönotisches Grundprincip. Arch. Hydrobiol., $49: 421-442$.

Trolzky (H.M.) \& Gregory (R.M.) 1974. - The effect of water flow manipulation below a hydroelectric power dam on the botton fauna of the Upper Kennebec River, Maine. Trans. Am. Fisch. Soc., 103: 318-324.

Viaud-Chauvet (M.) 1982. - Analyse des récoltes d'exuvies nymphates de Chironomides dáns les riviëres du Bassin du Tarn. Guide d'identification générique des exuvies nymphales de Diamesinae Prodiamesinate et Orthocladiinae de France. Relation ent re la structure des peuplements et la qualité des eaux. Thèse $3^{e}$ cycle, Hydrobiologie, Univ. P. Sabatier, Toulouse, France: $171 \mathrm{p}$.

Ward (J.V.). 1974. - A temperature-stressed stream ecosystem below a hypolinmial release mountain reservil. A roh. Hydrobiol., $2: 247-275$.

Ward (J.V.) \& Short (R.A.). 1978. - Macroinvertebrate community structure of four special lotic habitats in Colorado. U.S.A., Verh. Intermat. Verein. Limnol, 20 : 1382-1387.

Ward (J.V.) \& Stanford (J.A.). 1979. - Symposium summary and conclusions: pp 377-385. In : J.V. Ward \& J.A. Standord (Ed.) The ecology of regulded sireanis. New York. 